\title{
On weakly infinite-dimensional subspaces
}

\author{
by
}

Piet Borst (Amsterdam)

\begin{abstract}
We will construct weakly infinite-dimensional (in the sense of Y. Smirnov) spaces $X$ and $Y$ such that $Y$ contains $X$ topologically and $\operatorname{dim} Y=\omega_{0}$ and $\operatorname{dim} X=\omega_{0}+1$. Consequently, the subspace theorem does not hold for the transfinite dimension dim for weakly infinite-dimensional spaces.
\end{abstract}

Preliminaries. All the spaces considered are separable and metrizable. Let us first establish some notational conventions. As far as standard notions from general topology and dimension theory are concerned we mostly follow [E1] and [E2].

In particular, we note that the boundary of a subset $A$ of a space $X$ is denoted by $\operatorname{Fr} A$. We denote by $C$ the Cantor set, and by $\omega$ the collection of natural numbers. The first infinite ordinal is denoted by $\omega_{0}$.

1. Definitions. Let us start with some fundamental definitions.

1.1. Definition. A sequence $\left\{\left(A_{i}, B_{i}\right)\right\}_{i=1}^{n}$ of pairs of disjoint closed sets in a space $X$ is called inessential (resp. inessential on a subspace $F$ ) if we can find open sets $O_{i}$ (resp. $O_{i}$ open in $\left.F\right), i=1, \ldots, n$, such that

$$
A_{i} \subset O_{i} \subset \bar{O}_{i} \subset X-B_{i} \text { and } \bigcap_{i=1}^{n} \operatorname{Fr} O_{i}=\emptyset
$$

(resp. $\left.\left(\bigcap_{i=1}^{n} \operatorname{Fr} O_{i}\right) \cap F=\emptyset\right)$. Otherwise it is called essential (resp. essential on $F$ ).

1.2. Definition. A space $X$ is called weakly infinite-dimensional in the sense of Smirnov, abbreviated $S$-w.i.d., if for every sequence $\left\{\left(A_{i}, B_{i}\right)\right\}_{i=1}^{\infty}$ of pairs of disjoint closed sets in $X$ there exist open sets $O_{i}, i=1,2, \ldots$,

1991 Mathematics Subject Classification: Primary 54F45.

Key words and phrases: weakly infinite-dimensional, transfinite dimension. 
such that

$$
A_{i} \subset O_{i} \subset \bar{O}_{i} \subset X-B_{i} \text { and } \bigcap_{i=1}^{n} \operatorname{Fr} O_{i}=\emptyset \text { for some } n \text {. }
$$

In [B1] and [B2] we developed a transfinite extension of the covering dimension, dim. This dimension function classifies all weakly infinite-dimensional spaces. We also saw that the classification resulting from R. Pol's index $[\mathrm{P}]$ is equivalent and that the essential mappings defined by D. W. Henderson $[\mathrm{He}]$ give a classification which differs by at most 1 class from the one resulting from dim. We will see that the subspace theorem does not hold for the dimension dim. We prove this by constructing spaces $X$ and $Y$ such that $Y$ contains $X$ topologically and moreover $\operatorname{dim} X=\omega_{0}+1$ and $\operatorname{dim} Y=\omega_{0}$. We also see that this result is relevant to the characterization theorem.

To define the transfinite dimension function dim, we need the following notions:

Let $L$ be an arbitrary set. Fin $L$ denotes the collection of all finite, non-empty subsets of $L$. Let $M$ be a subset of Fin $L$.

For each $\sigma \in\{\emptyset\} \cup$ Fin $L$ we put

$$
M^{\sigma}=\{\tau \in \text { Fin } L: \sigma \cup \tau \in M \text { and } \sigma \cap \tau=\emptyset\} .
$$

$M^{\{a\}}$ is abbreviated as $M^{a}$.

1.3. Definition. Define the ordinal Ord $M$ as follows:

Ord $M=0 \quad$ iff $\quad M=\emptyset$,

$\operatorname{Ord} M \leq \alpha \quad$ iff $\quad$ for every $a \in L$, Ord $M^{a}<\alpha$,

$\operatorname{Ord} M=\alpha \quad$ iff $\quad \operatorname{Ord} M \leq \alpha$ and $\operatorname{Ord} M<\alpha$ is not true, and

Ord $M=\infty \quad$ iff $\quad$ Ord $M>\alpha$ for every ordinal $\alpha$.

Let $X$ be a normal space. Then we define

$$
L(X)=\{(A, B): A \cap B=\emptyset, A \text { and } B \text { are closed in } X\} .
$$

Moreover, let

$$
M_{L(X)}=\{\sigma \in \operatorname{Fin} L(X): \sigma \text { is essential }\} .
$$

We have the following equality:

1.4. Theorem. Let $X$ be a space. Then

$$
\operatorname{Ord} M_{L(X)} \leq n \quad \text { iff } \quad \operatorname{dim} X \leq n .
$$

This inspired our definition of transfinite dimension dim.

1.5. Definition. Let $X$ be a space. Then $\operatorname{dim} X=\operatorname{Ord} M_{L(X)}$.

We have seen in [B1] that

1.6. Theorem. For a space $X, \operatorname{dim} X$ exists iff $X$ is $S$-w.i.d. 
2. The subspace theorem. In [B1] we have proved the following subspace theorem on dim.

2.1. Theorem [B1, 3.1.6]. Let $F$ be a closed subset of a space $X$. Then $\operatorname{dim} F \leq \operatorname{dim} X$.

We also obtained the next result on open subspaces.

2.2. Proposition $[\mathrm{B} 1,3.5 .5]$. Let $Y$ be a space and let $X$ be an open subspace of $Y$ such that $\omega_{0} \leq \operatorname{dim} X<\infty$. Moreover, assume $\operatorname{dim}(Y-X)$ is finite. Then $\operatorname{dim} X=\operatorname{dim} Y$.

2.3. Example. Let us define Smirnov's spaces $S_{\alpha}$ for $\alpha<\omega_{1}$ :

$-S_{0}=\{0\}$,

$-S_{\alpha+1}=S_{\alpha} \times[-1,1]$

- if $\alpha$ is a limit then $S_{\alpha}=\omega\left(\bigoplus_{\beta<\alpha} S_{\beta}\right)$ (one-point compactification).

It is well known that each $S_{\alpha}$ is S-w.i.d. Moreover, $\operatorname{dim} S_{\alpha}=\alpha$. The proof is completely analogous to $[\mathrm{B} 2 ; 4.1 .11]$.

The discrete sum $Z=\bigoplus_{n<\omega_{0}} S_{n}$ is not S-w.i.d. but it is topologically contained in $S_{\omega_{0}}$. Therefore we see that a subspace $X$ of an S-w.i.d. space $Y$ need not be S-w.i.d. itself.

So we have $Z \subset S_{\omega_{0}}, \operatorname{dim} Z=\infty$ and $\operatorname{dim} S_{\omega_{0}}=\omega_{0}$.

The natural question arises whether for every S-w.i.d. space $Y$ and every S-w.i.d. subspace $X$ of $Y$ we have $\operatorname{dim} X \leq \operatorname{dim} Y$.

2.4. Remark. For the transfinite dimension function Ind, which has very similar properties to dim and classifies a similar category of infinitedimensional spaces, L. A. Lyuksemburg proved

2.5. TheOrem $[\mathrm{L}]$. Let $Y$ be a metric space and let $X$ be a subspace of $Y$ such that both Ind $X$ and $\operatorname{Ind} Y$ exist. Then $\operatorname{Ind} X \leq \operatorname{Ind} Y$.

Thus for Ind the subspace theorem holds.

We will answer our question in the negative by constructing a counterexample.

3. The counterexample. We will define S-w.i.d. spaces $X$ and $Y$ such that $X \subset Y \subset S_{\omega_{0}+1}, \operatorname{dim} X=\omega_{0}+1$ and $\operatorname{dim} Y=\omega_{0}$.

Put $T_{n}=S_{n} \times I$ for $n=1,2, \ldots$ and let

$$
S_{\omega_{0}+1}=S_{\omega_{0}} \times I=\{p\} \times I \cup \bigoplus_{n<\omega_{0}}\left(S_{n} \times I\right)=\{p\} \times I \cup \bigoplus_{n<\omega_{0}} T_{n} .
$$

For the construction of $Y$, for each $n=1,2, \ldots$ express the interval $I=[-1,1]$ as $I=\bigcup_{m=-n+1}^{n} K_{n m}$ where $K_{n m}=[(m-1) / n, m / n]$. Fix some $n$ and some $m \in\{-n+1, \ldots, n\}$. We may define $O_{n m}^{i}, i=1,2, \ldots$, 
as open intervals in $I$ within $K_{n m}$ such that $O_{n m}^{i} \cap O_{n m}^{j}=\emptyset$ for $i \neq j$ and $i, j=1,2, \ldots$ Let us also define

$$
F_{n}^{i}=\chi_{j=1}^{n}[-1 /(i+1), 1 /(i+1)] \subset S_{n}
$$

for each $n=1,2, \ldots$ and $i=1,2, \ldots$ For each $n$ let

$$
\begin{aligned}
Y_{n}=\left\{(x, y) \in S_{n} \times I: y \notin O_{n m}^{i}\right. \\
\quad \text { for every } m=-n+1, \ldots, n \text { and every } i=1,2, \ldots\} \\
\cup\left\{(x, y) \in S_{n} \times I: y \in O_{n m}^{i} \text { and } x \in F_{n}^{i}\right. \\
\quad \text { for some } m=-n+1, \ldots, n \text { and some } i=1,2, \ldots\} .
\end{aligned}
$$

Clearly, $Y_{n} \subset T_{n}$.

We define $Y=\{p\} \times I \cup \bigoplus_{n<\omega_{0}} Y_{n}$. Then $Y$ is closed subspace of $S_{\omega_{0}+1}$ and hence a compact metric space.

Now let us construct the subspace $X$ of $Y$. Set

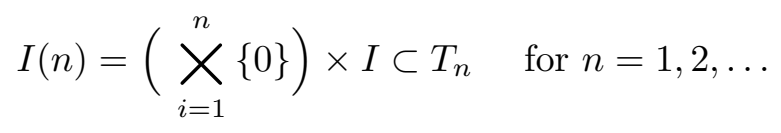

Note that also $I(n) \subset Y_{n}$ for each $n=1,2, \ldots$ Define $X_{n}=Y_{n}-I(n)$ and $X=\{p\} \times I \cup \bigoplus_{n<\omega_{0}} X_{n}$. Clearly we have $X \subset Y \subset S_{\omega_{0}+1}$.

We will prove that $\operatorname{dim} Y=\omega_{0}$ but its subspace $X$ is $\mathrm{S}$-w.i.d. and has dimension $\operatorname{dim} X>\omega_{0}$.

3.1. Claim. $\operatorname{dim} Y=\omega_{0}$.

Clearly, $\operatorname{dim} Y \geq \omega_{0}$ since $Y$ contains closed subspaces of arbitrary large finite dimension. To show $\operatorname{dim} Y \leq \omega_{0}$, let $\left(A_{0}, B_{0}\right)$ be a pair of disjoint closed subsets in $Y$ (in other words, $\left(A_{0}, B_{0}\right) \in L(Y)$ ). We prove that there exists some finite $n_{1}$ such that

$$
\operatorname{Ord} M_{L(Y)}^{\left(A_{0}, B_{0}\right)}<n_{1}
$$

Consider $\{p\} \times I$ in $Y$ as the unit interval $[-1,1]$. There exists some $k$ such that for all the subintervals $K_{k m}=[(m-1) / k, m / k], m=-k+1, \ldots, k$, we have

$$
\text { if } \quad K_{k m} \cap A_{0} \neq \emptyset \text { then }\left(K_{k, m-1} \cup K_{k m} \cup K_{k, m+1}\right) \cap B_{0}=\emptyset .
$$

For each $m$ we can find some $n(m)$ such that for each $n \geq n(m)$ we have

$$
\begin{array}{llll}
\text { if } & K_{k m} \cap A_{0}=\emptyset & \text { then } \quad\left(S_{n} \times K_{k m}\right) \cap A_{0}=\emptyset \quad \text { and } \\
\text { if } & K_{k m} \cap B_{0}=\emptyset & \text { then } \quad\left(S_{n} \times K_{k m}\right) \cap B_{0}=\emptyset .
\end{array}
$$

Put $n_{1}=\max \{2 k, n(m): m=-k+1, \ldots, k\}$. We prove that $n_{1}$ is as required. Take $\left\{\left(A_{i}, B_{i}\right)\right\}_{i=0}^{n_{1}} \in \operatorname{Fin} L(Y)$. If we prove

$$
\left\{\left(A_{i}, B_{i}\right)\right\}_{i=0}^{n_{1}} \text { is inessential, }
$$




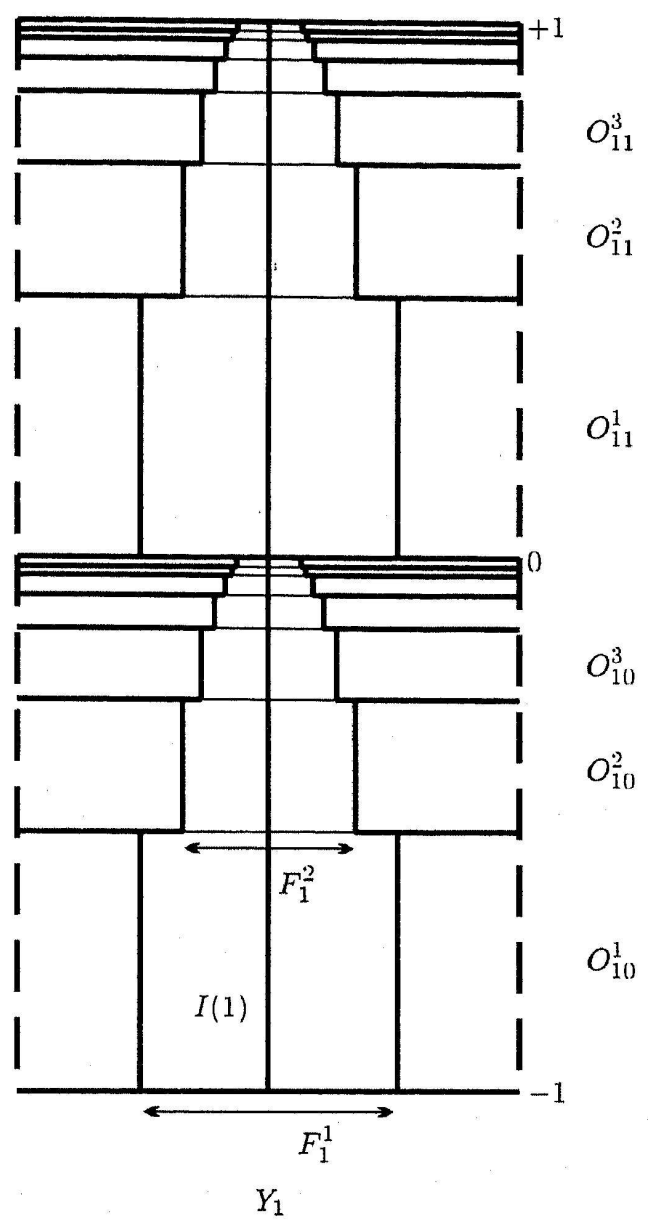

we are done. Let $G=\bigoplus_{n=0}^{n_{1}-1} Y_{n}$. Then $\operatorname{dim} G \leq n_{1}$; hence by the theorem on partitions $[\mathrm{E} 2 ; 3.2 .6]$ we can find open sets $U_{i}$ in $G, i=0, \ldots, n_{1}$, such that

$$
A_{i} \cap G \subset U_{i} \subset \bar{U}_{i} \subset G-B_{i} \text { and } \bigcap_{i=0}^{n_{1}} \operatorname{Fr} U_{i}=\emptyset .
$$

Now consider $F=Y-G=\{p\} \times I \cup \bigoplus_{n_{1} \leq n<\omega_{0}} Y_{n}$. Since $\operatorname{dim}\{p\} \times I=1$ we can find open sets $V_{i}^{\prime}$ in $F, i=0, \ldots, n_{1}$, such that

$$
A_{i} \cap F \subset V_{i}^{\prime} \subset \bar{V}_{i}^{\prime} \subset F-B_{i} \text { and } \bigcap_{i=0}^{n_{1}} \operatorname{Fr} V_{i}^{\prime} \cap\{p\} \times I=\emptyset .
$$

There is some $n_{2} \geq n_{1}$ such that

$$
\bigcap_{i=0}^{n_{1}} \operatorname{Fr} V_{i}^{\prime} \cap\left(\{p\} \times I \cup \bigoplus_{n_{2}<n<\omega_{0}} Y_{n}\right)=\emptyset .
$$


For $i=0, \ldots, n$, let $V_{i}=V_{i}^{\prime} \cap\left(\{p\} \times I \cup \bigoplus_{n_{2}<n<\omega_{0}} Y_{n}\right)$. Then

$$
\bigcap_{i=0}^{n_{1}} \operatorname{Fr} V_{i}=\emptyset \text {. }
$$

For each $n=n_{1}, \ldots, n_{2}$ consider the set $Y_{n}$ and $\left\{\left(A_{i}, B_{i}\right)\right\}_{i=0}^{n_{1}}$ restricted to the subspace $Y_{n}$. The space $Y_{n}$ is compact, $\left(A_{1}, B_{1}\right)$ is a pair of disjoint closed sets and for every $m=-n+1, \ldots, n$ we have

$$
\lim _{i \rightarrow \infty} \operatorname{diam}\left(F_{n}^{i} \times O_{n m}^{i}\right)=0 .
$$

Since $n \geq n_{1} \geq 2 k$, for every $l=-k+1, \ldots, k$ there exists an $m(l) \in$ $\{-n+1, \ldots, n\}$ such that $K_{n m(l)} \subset K_{k l}$.

We can find some $i_{0}$ such that for each $m(l)$, where $l=-k+1, \ldots, k$, we have

$$
\left(F_{n}^{i_{0}} \times \bar{O}_{n m(l)}^{i_{0}}\right) \cap A_{1}=\emptyset \quad \text { or } \quad\left(F_{n}^{i_{0}} \times \bar{O}_{n m(l)}^{i_{0}}\right) \cap B_{1}=\emptyset .
$$

Let $l_{1}, \ldots, l_{p}$ be all $l,-k+1 \leq l \leq k$, such that $K_{k l} \cap A_{0}=\emptyset$ and $K_{k l} \cap B_{0}$ $=\emptyset$. Then by our choice of $n_{1}$ we have for $n=n_{1}, \ldots, n_{2}, j=1, \ldots, p$

$$
\left(S_{n} \times K_{k l_{j}}\right) \cap A_{0}=\emptyset \quad \text { and } \quad\left(S_{n} \times K_{k l_{j}}\right) \cap B_{0}=\emptyset .
$$

Since $\bar{O}_{n m\left(l_{j}\right)}^{i_{0}} \subset K_{n m\left(l_{j}\right)} \subset K_{k l}$, (3.1.2) and the construction of $Y_{n}$ show that the set

$$
P_{n}=\bigcup_{j=1}^{p}\left(F_{n}^{i_{0}} \times \bar{O}_{n m\left(l_{j}\right)}^{i_{0}}\right)
$$

forms a partition of $Y_{n}$ between $A_{0} \cap Y_{n}$ and $B_{0} \cap Y_{n}$. Consequently, we can find some open set $V_{0}^{n}$ in $Y_{n}$ such that

$$
A_{0} \cap Y_{n} \subset V_{0}^{n} \subset \bar{V}_{0}^{n} \subset Y_{n}-B_{0} \text { and } \operatorname{Fr} V_{0}^{n} \subset P_{n} .
$$

By virtue of (3.1.6) and the normality of $Y$ we can find an open set $V_{1}^{n}$ such that

$$
A_{1} \cap Y_{n} \subset V_{1}^{n} \subset \bar{V}_{1}^{n} \subset Y_{n}-B_{1} \text { and } \operatorname{Fr} V_{1}^{n} \cap P_{n}=\emptyset .
$$

Then, by (3.1.7), also $\operatorname{Fr} V_{0}^{n} \cap \operatorname{Fr} V_{1}^{n}=\emptyset$.

Now for $i=2, \ldots, n_{1}$ let $V_{i}^{n}$ be an open subset of $Y_{n}$ such that

$$
A_{i} \cap Y_{n} \subset V_{i}^{n} \subset \bar{V}_{i}^{n} \subset Y_{n}-B_{i} .
$$

Then also $\bigcap_{i=0}^{n_{1}} \operatorname{Fr} V_{i}^{n}=\emptyset$. For $i=0, \ldots, n_{1}$ let

$$
O_{i}=U_{i} \cup V_{i} \cup\left(\bigcup_{n=n_{1}}^{n_{2}} V_{i}^{n}\right) .
$$

Then each $O_{i}, i=0, \ldots, n_{1}$, is an open set in $Y$ such that according to (3.1.4), (3.1.5) and (3.1.9) we have

$$
A_{i} \subset O_{i} \subset \bar{O}_{i} \subset Y-B_{i} .
$$


Then also $\bigcap_{i=0}^{n_{1}} \operatorname{Fr} O_{i}=\emptyset$. Consequently, we have proven (3.1.3) and we are done.

3.2. Claim. $\operatorname{dim} X \geq \omega_{0}+1$.

For this, consider $A=S_{\omega_{0}} \times[-1,-1 / 2]$ and $B=S_{\omega_{0}} \times[1 / 2,1]$. Clearly, $(A, B)$ forms a pair of disjoint closed sets in $S_{\omega_{0}+1}=S_{\omega_{0}} \times I$. Let $A_{0}=A \cap X$ and $B_{0}=B \cap X$. We will prove our claim by showing that

$$
\operatorname{Ord} M_{L(X)}^{\left(A_{0}, B_{0}\right)} \geq n \quad \text { for each } n<\omega_{0} .
$$

For this, fix some finite $n$ and consider the subspace $T_{n+1}=S_{n+1} \times I$ within $S_{\omega_{0}+1}$. Observe that $C=S_{n+1} \times\{-1\} \subset A$ and $D=S_{n+1} \times\{1\} \subset B$ can be considered as opposite faces of the cube $T_{n+1}$. We have

$$
S_{n+1}=\left\{x=\left(x_{1}, \ldots, x_{n+1}\right): x_{i} \in[-1,1], i=1, \ldots, n+1\right\} .
$$

For $i=1, \ldots, n+1$ put $C_{i}=\left\{x \in S_{n+1}: x_{i}=-1\right\}$ and $D_{i}=\left\{x \in S_{n+1}\right.$ : $\left.x_{i}=1\right\}$. The pairs $\left(C_{i} \times I, D_{i} \times I\right)$ also form pairs of opposite faces of the cube $T_{n+1}$.

In addition, define

$$
\begin{aligned}
F_{i} & =\left\{x \in S_{n+1}: x_{i} \leq 0 \text { and } x_{j} \geq x_{i} \text { for } j \neq i\right\}, \\
G_{i} & =\left\{x \in S_{n+1}: x_{i} \geq 0 \text { and } x_{j} \leq x_{i} \text { for } j \neq i\right\} .
\end{aligned}
$$

Clearly, $C_{i} \subset F_{i}$ and $D_{i} \subset G_{i}$ for $i=1, \ldots, n+1$. Observe that $F_{i} \cap G_{i}=$ $\{(0, \ldots, 0)\}$. Consequently, $F_{i} \times I \cap G_{i} \times I=\{(0, \ldots, 0)\} \times I=I(n+1)$, so that by the construction of $X$ we have $F_{i} \times I \cap G_{i} \times I \cap X=\emptyset$.

Put $A_{i}=F_{i} \times I \cap X$ and $B_{i}=G_{i} \times I \cap X$ for $i=1, \ldots, n+1$. It is sufficient to prove that

$$
\left\{\left(A_{i}, B_{i}\right)\right\}_{i=0}^{n} \text { is essential on the subspace } X_{n+1} \text {. }
$$

Assume the contrary. Then there are open sets $U_{i}, i=0, \ldots, n$, in $X_{n+1}$ such that

$$
A_{i} \cap X_{n+1} \subset U_{i} \subset \bar{U}_{i} \subset X_{n+1}-B_{i} \quad \text { for } i=0, \ldots, n
$$

and $\bigcap_{i=0}^{n} \operatorname{Fr} U_{i}=\emptyset$. According to [E2; 1.2.9], we can extend the $U_{i}$ to open sets $V_{i}$ in $T_{n+1}$, for $i=0, \ldots, n$, such that

$$
\begin{aligned}
& {\left[\left(F_{i}-\{(0, \ldots, 0)\}\right) \times I\right] \cap T_{n+1} \subset V_{i} \subset \bar{V}_{i} } \\
& \subset T_{n+1}-\left[\left(G_{i}-\{(0, \ldots, 0)\}\right) \times I\right] \quad \text { for } i=1, \ldots, n
\end{aligned}
$$

and

$$
C \subset V_{0} \subset \bar{V}_{0} \subset T_{n+1}-D, \quad \operatorname{Fr} V_{i} \cap X_{n+1}=\operatorname{Fr} U_{i} \quad \text { for } i=0, \ldots, n
$$

so that $\bigcap_{i=0}^{n} \operatorname{Fr} V_{i} \cap X_{n+1}=\emptyset$. For brevity we put $E=\bigcap_{i=0}^{n} \operatorname{Fr} V_{i}$ so that

$$
E \cap X_{n+1}=\emptyset \text {. }
$$


Let $O=\left\{x \in S_{n+1}: x_{n+1}<-3 / 4\right\}$. Then

$$
C_{n+1} \subset O \subset \bar{O} \subset S_{n+1}-D_{n+1} .
$$

Thus $O \times I$ is an open set in $S_{n+1} \times I=T_{n+1}$ such that

$$
C_{n+1} \times I \subset O \times I \subset \bar{O} \times I \subset T_{n+1}-D_{n+1} \times I .
$$

According to the construction of $Y$ and $X$ and the definition of $O$ we have

$$
(\bar{O} \times I)-X=(\bar{O} \times I)-Y=\bar{O} \times \bigcup_{\substack{i=1,2, \ldots \\ m=-n+1, \ldots, n}} O_{n m}^{i} .
$$

Statement (3.2.4) then yields

$$
(\bar{O} \times I) \cap E \subset \bar{O} \times \bigcup_{\substack{i=1,2, \ldots \\ m=-n+1, \ldots, n}} O_{n m}^{i} .
$$

By the compactness of $E$ we may assume

$$
(\bar{O} \times I) \cap E \subset \bar{O} \times \bigcup_{j=1}^{k} O_{n m_{j}}^{i_{j}} .
$$

Moreover, by (3.2.3) we have

$$
E \subset\left[\left(S_{n+1}-\bigcup_{i=1}^{n}\left(F_{i} \cup G_{i}\right)\right) \cup\{(0, \ldots, 0)\}\right] \times I .
$$

For $j=1, \ldots, k$ define

$$
W_{j}=S_{n+1}-\left(\left(\bigcup_{i=1}^{n} F_{i} \cup G_{i}\right) \cup G_{n+1} \cup F_{n}^{i_{j}}\right) .
$$

Then (3.2.6), $\bar{O} \cap G_{n+1}=\emptyset, \bar{O} \cap F_{n}^{i_{j}}=\emptyset$ and $(0, \ldots, 0) \notin \bar{O}$ yield

$$
E \cap(\bar{O} \times I) \subset W_{j} \times I \quad \text { for each } j=1, \ldots, k .
$$

Combining this and (3.2.5) we obtain

$$
E \cap(\bar{O} \times I) \subset \bigcup_{j=1}^{k}\left(W_{j} \times O_{n m_{j}}^{i_{j}}\right) .
$$

From the definition (3.2.7) it is clear that for $j=1, \ldots, k$

$$
\operatorname{Fr} W_{j} \subset \bigcup_{i=1}^{n}\left(F_{i} \cup G_{i}\right) \cup F_{n}^{i_{j}} .
$$

Together with (3.2.4) and (3.2.6) this gives us

$$
E \cap\left(\operatorname{Fr} W_{j} \times O_{n m_{j}}^{i_{j}}\right)=\emptyset .
$$




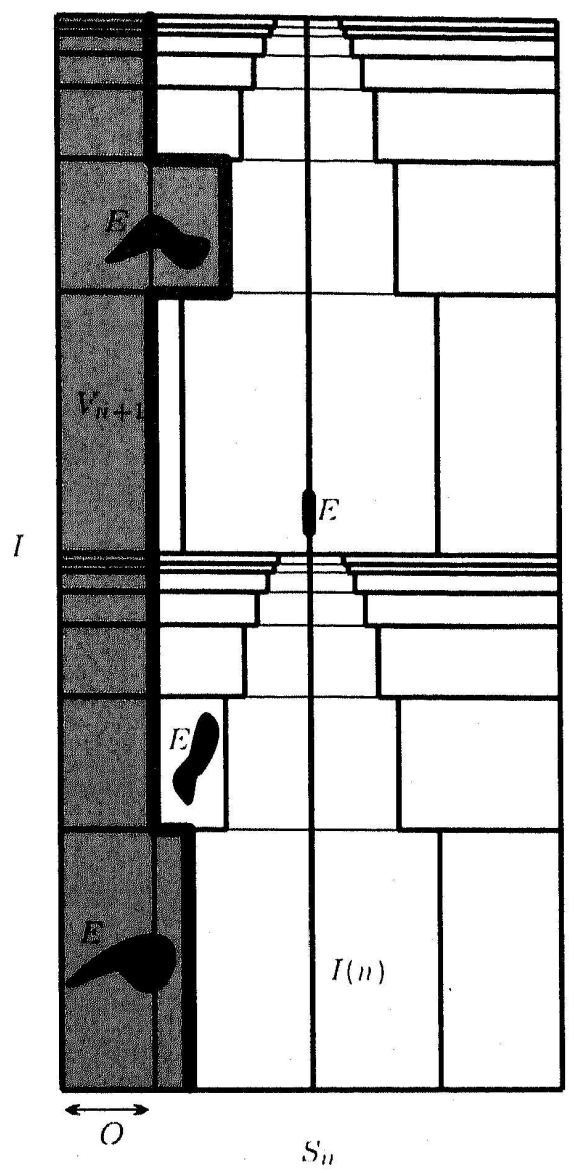

Now put

$$
V_{n+1}=\bigcup_{j=1}^{k}\left(W_{j} \times O_{n m_{j}}^{i_{j}}\right) \cup(O \times I) .
$$

Then according to (3.2.8) and (3.2.9)

$$
\emptyset=\operatorname{Fr} V_{n+1} \cap E=\operatorname{Fr} V_{n+1} \cap \bigcap_{i=0}^{n} \operatorname{Fr} V_{i}=\bigcap_{i=0}^{n+1} \operatorname{Fr} V_{i} .
$$

By the definition of $O$ and since $W_{j} \cap D_{n+1}=\emptyset$ (since $D_{n+1} \subset G_{n+1}$ ) we have

$$
C_{n+1} \times I \subset V_{n+1} \subset \bar{V}_{n+1} \subset T_{n+1}-D_{n+1} \times I .
$$

We have already seen that $C \subset V_{0} \subset \bar{V}_{0} \subset T_{n+1}-D$. However, the sequence $\left\{(C, D),\left(C_{1} \times I, D_{1} \times I\right), \ldots,\left(C_{n+1} \times I, D_{n+1} \times I\right)\right\}$ contains the pairs of opposite faces of the $(n+2)$-dimensional cube $T_{n+1}$ and hence is essential 
[E2; 1.8.1]. We reach a contradiction. Therefore assertion (3.2.1) is proven and we are done.

For the proof of our next claim we need the following proposition [B1; $5.3 .2]$.

3.3. Proposition. Suppose that for a space $X$ there exists a closed subspace $G$ such that $\operatorname{dim} G$ is finite and for each $F$ closed in $X$ such that $G \cap F=\emptyset, \operatorname{dim} F$ is finite. Then $\operatorname{dim} X \leq \omega_{0}+\operatorname{dim} G$.

3.4. Claim. $\operatorname{dim} X \leq \omega_{0}+1$ and consequently, $X$ is S-w.i.d.

Indeed, we apply Proposition 3.3. Let $G=\{p\} \times I$. Then $\operatorname{dim} G=1$. Moreover, for each $F$ closed in $X$ and disjoint from $G$ we have $F \subset$ $\bigoplus_{m=0}^{n} X_{m}$ for some $n$ so that $\operatorname{dim} F \leq n+1<\omega_{0}$. Hence $\operatorname{dim} X \leq$ $\omega_{0}+\operatorname{dim} G=\omega_{0}+1$. By Theorem 1.6 we see that $X$ is S-w.i.d.

We conclude that $X \subset Y$ and $\operatorname{dim} Y=\omega_{0}$, but for $X$ we have $\operatorname{dim} X=$ $\omega_{0}+1$.

4. Relation with the characterization theorem. In [B2] we proved the following theorem:

4.1. Theorem [B2; 4.2.1]. Let $X$ be a locally compact space and $\alpha<\omega_{1}$. Then $\operatorname{dim} X \geq \alpha$ iff $X \times C$ admits an essential map onto $J^{\alpha}$.

The transfinite cubes $J^{\alpha}$ and the concept of essential mappings to $J^{\alpha}$ are defined by D. W. Henderson [He]. The local compactness restriction follows from the use of the following product theorem in the proof.

4.2. Theorem [B1; 3.5.7]. Let $X$ be a locally compact space. Then $\operatorname{dim} X=\operatorname{dim} X \times C$.

In $[\mathrm{Ch}] \mathrm{V}$. A. Chatyrko proved the following compactification theorem.

4.3. Theorem [Ch]. Let $X$ be an S-w.i.d. space. Then:

(1) $\operatorname{dim} X=\operatorname{dim} \beta X$.

(2) We can find a compact metric space $Y$ such that $Y$ contains $X$ topologically and $\operatorname{dim} Y \leq \operatorname{dim} X$.

Combining results $2.1,4.2$ and 4.3 he observes that using the compactification $Y$ of $X$ we can almost prove $\operatorname{dim} X=\operatorname{dim} X \times C$ without the requirement of local compactness:

$$
\operatorname{dim} X \times C \stackrel{(2.1)}{\geq} \operatorname{dim} X \stackrel{(4.3)}{\geq} \operatorname{dim} Y \stackrel{(4.2)}{=} \operatorname{dim} Y \times C \stackrel{?}{\geq} \operatorname{dim} X \times C .
$$

We only need the subspace theorem and we are done.

In this regard, but also considering the general requirements for a dimension function, it is a pity the subspace theorem does not generally hold. 
The following question remains unanswered.

4.4. Question. Can Theorems 4.1 and 4.2 be extended beyond the class of locally compact spaces?

The author is indebted to V. A. Chatyrko and J. van Mill for their encouragement and advice.

\section{References}

[B1] P. Borst, Classification of weakly infinite-dimensional spaces. Part I: A transfinite extension of the covering dimension, Fund. Math. 130 (1988), 1-25.

[B2] -, Classification of weakly infinite-dimensional spaces. Part II: Essential mappings, ibid., 73-99.

[Ch] V. A. Chatyrko, On the transfinite dimension dim, to appear.

[E1] R. Engelking, General Topology, PWN, Warszawa 1977.

[E2] - Dimension Theory, PWN, Warszawa 1978.

[He] D. W. Henderson, A lower bound for transfinite dimension, Fund. Math. 63 (1968), 167-173.

[L] L. A. Lyuksemburg, On transfinite inductive dimensions, Soviet Math. Dokl. 14 (1973), 388-393.

[P] R. Pol, On classification of weakly infinite-dimensional compacta, Fund. Math. 116 (1983), 169-188.

DEPARTMENT OF MATHEMATICS

FREE UNIVERSITY

DE BOELELAAN 1081

1081 HV AMSTERDAM, THE NETHERLANDS

Received 12 November 1990;

in revised form 25 February 1991 and 24 July 1991 